\title{
Anterior cervical discectomy and fusion with ROI-C peek cage: cervical alignment and patient outcomes
}

\author{
Giovanni Grasso • Filippo Giambartino • \\ Giovanni Tomasello • Gerardo Iacopino
}

Received: 28 August 2014/Revised: 3 September 2014/ Accepted: 3 September 2014

(C) Springer-Verlag Berlin Heidelberg 2014

\begin{abstract}
Objective The intersomatic cage ROI-C one is a new promising system in anterior cervical discectomy with subsequent fusion.

Methods Patients were studied prospectively. ROI-C cages were used in consecutive 32 patients. Intraoperative parameters, clinical features and dysphagia scores were recorded. Radiographs evaluated the height of intervertebral space, the cervical Cobb angle and implant positioning. Data were collected on the last day of hospital stay, at 6 weeks, at 3, 6, 12 and 24 months.

Results A total of 32 cages were implanted. One patient had transient dysphagia. The intervertebral height and the cervical Cobb angle were significantly improved at 24 months follow-up $(P<0.05)$. Compared to pre-operatively, visual analog scale pain score and neck pain and disability scale were reduced at 1-month follow-up without change during subsequent follow-up.

Conclusions This is the first prospective study on ROI-C cages. Although this is a preliminary assessment, the ROI$\mathrm{C}$ cage may represent an excellent alternative to other devices or simple bone graft.
\end{abstract}

G. Grasso $(\bowtie) \cdot$ G. Iacopino

Department of Experimental Biomedicine and Clinical

Neurosciences, School of Medicine, Neurosurgical Clinic,

University of Palermo, Via del Vespro 129, 90100 Palermo, Italy

e-mail: giovanni.grasso@unipa.it

F. Giambartino

Department of Anesthesiology, School of Medicine, University

of Palermo, Palermo, Italy

G. Tomasello

Department of Surgery, School of Medicine, University of

Palermo, Palermo, Italy
Keywords Anterior cervical discectomy - Cervical disc herniation $\cdot$ ROI-C $\cdot$ Vertebral fusion

\section{Introduction}

Cervical discectomy is one of the most commonly and rapidly growing procedures done to rapidly restore radicular and medullar compression [1]. Since fusion procedures are widely accepted, research and development has concentrated on improving and simplifying portions of this procedure in hopes that it would improve outcomes. Although a large number of technical and biomechanical advances have led to an array of available options for fusions, for many of these devices, there are few objective evaluations in the literature. Decision making among these options can be bewildering for the experienced surgeon as well as for the novice.

The ROI-C cage (LDR Médical- France) is one of the devices that have been developed to increase cervical spine stability during the early post-operative period. In addition to immediate stabilization, use of these types of devices allows avoidance of anterior plating and iliac crest bone grafting. These cages are increasingly used in anterior cervical discectomy with fusion (ACDF), but detailed, relatively objective assessments are not available. We present our experience in a medium-term outcome assessment.

\section{Methods}

Patient populations and indications

Between January 2009 and January 2011, we performed anterior cervical discectomy and fusions with the ROI-C 
Table 1 Summary of demographic data

\begin{tabular}{llll}
\hline Variable & Male & Female & Total \\
\hline No. cases & 18 & 14 & 32 \\
Age (years) mean & 60.91 & 58.25 & 59.80 \\
Range & $55-71$ & $47-69$ & $47-71$ \\
\hline
\end{tabular}

Table 2 Patient selection criteria

Criteria
Inclusion
Age $18-70$ years
Symptomatic cervical disc disease (with radicular pain and/or
neurologic deficit. With CT or MRI comfort)
Exclusion
Previous surgery at the same level
Fused level adjacent to the interested level
Medical condition(s) that would represent an increase in surgical
risk
Patients refusing consent
Systemic or local infection
Active rheumatoid arthritis
Known allergy to the materials contained in the device

cage for the treatment of cervical disc herniation or spondylosis causing radiculopathy on 32 consecutive patients. The study group had a total of 32 cages implanted. Patients had not experienced any cervical spine surgery before this operation. Table 1 summarizes patients' characteristics.

Criteria for inclusion were unremitting radicular arm pain, with or without neck pain, and/or a neurological deficit that correlated with appropriate level and side neural compression on magnetic resonance imaging (MRI) or computed tomography (CT). Patients who presented with other spinal degenerative conditions, such as stenosis or arthritis, were not excluded as long as the diagnosis indicated that the primary cause for complaints was clinically consistent with nerve root compression. Patients were also not excluded because of age, sex, compensation claims, diabetes, obesity or other medical conditions that would not preclude surgery in general (Table 2 ).

\section{The ROI-C cage}

The ROI-C cage is a cervical intersomatic cage made by polyetheretherketone (PEEK) optima (radiolucent) using the VerteBRIDGE ${ }^{\mathrm{TM}}$ double anchoring system to obtain an intervertebral fusion. This cage is designed specifically for implantation into the cervical disc space after discectomy. It enables the filling of a graft: either autologous fusion or bone substitute. It has been tailored to have zero profile, with no hardware protruding outside of the vertebral bodies.

Self-locking plates designed for initial and long-term stability. The self-guided, curved plating is delivered in the plane of the disc through a direct anterior approach. Figure 1 depicts the cage.

The cage is simple and intuitive, dedicated instruments are very few and the learning curve is almost brief. Stabilization appears to be immediately afforded following implantation, and the double anchoring system avoids effectively the use of a post-operative collar.

\section{Surgical procedure}

The surgical approach was consistent throughout the study. All the surgical procedures were performed by the first author (GG). The patients were placed supine and fluoroscopic images were obtained in anterior-posterior (AP) and lateral planes to determine the level of the diseased disc. A standard right-sided approach was undertaken to access the anterior cervical spine. A complete anterior discectomy was performed. When indicated, the posterior longitudinal ligament was released to allow for excision of extruded disc material and/or to determine the appropriate intervertebral disc height. Only the cartilaginous portion of the vertebral endplate was removed. Correct level distraction and ideal cage choice was performed according to lateral fluoroscopically assisted trialing, to obtain a satisfactory height and AP diameter. After the midline was determined, the cage trial was used. The trial was, than, removed and the appropriate-sized final cage endplates was inserted to an adequate depth under lateral fluoroscopic guidance. Following this, AP and lateral fluoroscopy were performed to confirm the appropriate positioning and size of the device. In all cases, cadaveric bony tissue was used to fill the cage to facilitate the bony integration process. Thereafter, the dedicated double anchoring system was inserted in the up and down vertebral body to obtain an intervertebral strength positioning.

\section{Outcome}

Post-operatively, cervical collar was not used, except in some patients who asked the externals device as "comfort". Usually, following 5 weeks, patients were allowed to return to all normal activities except contact sports on a permanent basis. The average hospitalization time was 1.95 days (range $1-4$ days).

During follow-up, clinical and radiographic data were collected on the last day of hospital stay, at 6 weeks, at 3, 6, 12 and at 24 months. Complications were recorded as implant related, surgery related, or general (not directly implant or surgery related). All the patients were included 

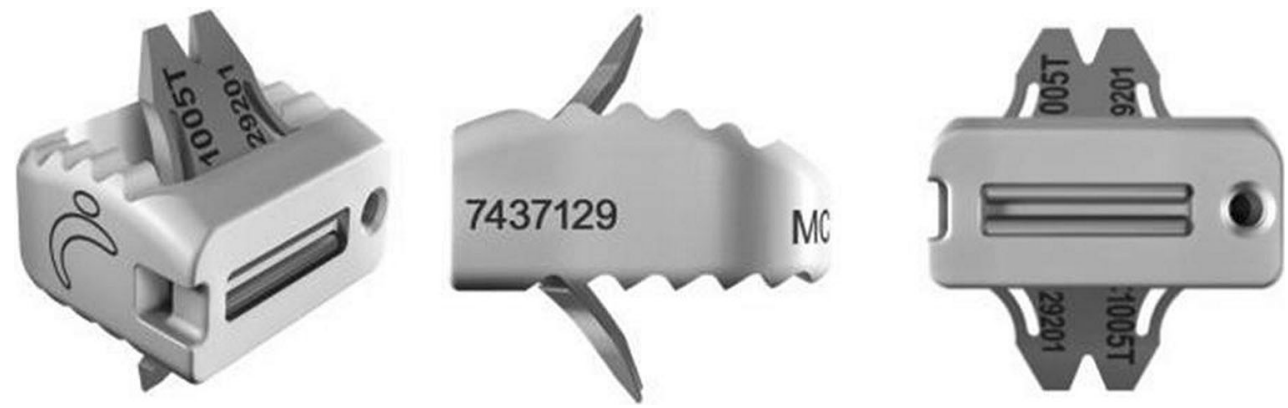

Fig. 1 The ROI-C cervical cage with VerteBRIDGE plating technology in different views

in the clinical and radiographic evaluations at each followup time point. Clinical examination included measurement of neck and radicular arm pain on a visual analog scale (VAS) of $0-100$, with 0 representing no pain and 100 representing severe pain [2,3], and assessment of functional outcome using the neck pain and disability scale (NPAD-D), expressed as a percentage ranging between 0 and $100 \%[4,5]$. Dysphagia-related symptoms were graded depending on the patient's state as none (no episodes of swallowing problems), mild (rare episodes of dysphagia), moderate (occasional swallowing difficulty with specific food), and severe (frequent difficult swallowing with majority of food) [6]. Additionally, the amount of pain (VAS 0-100) and the duration of dysphagia-related symptoms were recorded. A general surgeon, blinded of the study, assessed this issue.

A radiologist independently evaluated all images including plain radiographs and lateral flexion/extension radiographs. At the day of discharge and at each follow-up visit, plain radiographs (AP and lateral views) were used to detect device failure, including segmental collapse, caused by device subsidence. A device penetration into the adjacent endplates of more than $2 \mathrm{~mm}$ was defined as segmental collapse [7]. According to the literature [8], fusion was defined as an absence of radiolucency, absence of bone sclerosis, and evidence of bridging trabecular bone within the fusion area. Based on the lateral radiographs of the cervical spine at the neutral position, the sagittal alignment of the cervical spine was evaluated using the Cobb angle between $\mathrm{C} 2$ and $\mathrm{C} 7$. Furthermore, before and after operation the height of intervertebral space was measured.

\section{Statistical analysis}

We computed means and SDs for continuous data (VAS, NPAD-D scores, disc height and Cobb angle). We determined differences in VAS and NPAD-D scores between pre-operative and post-operative time points and during further follow-up using the $t$ test for paired samples if a normality test was passed or a Wilcoxon signed-rank test if
Table 3 Surgically treated levels

\begin{tabular}{lcll}
\hline Level & Total number & Male & Female \\
\hline C3-C4 & 4 & 4 & 3 \\
C4-C5 & 6 & 5 & 2 \\
C5-C6 & 15 & 7 & 5 \\
C6-C7 & 7 & 2 & 4 \\
\hline
\end{tabular}

a normality test was failed. Intra-observer variability for radiographic evaluation was determined using kappa statistics. SPSS 16.0 for Windows (Chicago, IL) software was used for all analyses.

\section{Results}

Patient population

Thirty-two patients (18 men and 14 women; mean age 59.80 years, range $47-71$ years) underwent implantation of a ROI-C cage. All the patients had 24 months follow-up.

A diagnosis of radiculopathy and/or myelopathy was established in all patients before surgery. Levels treated are illustrated in Table 3. In all the cases, cadaveric bony tissue was used to fill the cage.

\section{Outcomes}

In the early post-operative period, 2 of 32 patients $(6.25 \%)$ complained about minor dysphagia (VAS score $1.8 \pm 0.8$ ) with symptom duration of $7 \pm 3$ days. At 6 weeks' followup, the number of patients complaining of minor dysphagia was reduced to 1 of 2 patients. Only one patient complained about minor dysphagia (VAS score 1.6) at 12 months follow-up with a complete resolution at 24 months follow-up. According to a well-recognized score [6], there was no moderate or severe dysphagia detectable during follow-up. Overall, we observed no device-related complications. In particular, we observed no 
Fig. 2 Line graphs showing a the height of intervertebral space and $\mathbf{b}$ the cervical Cobb angle pre-operatively and during follow-up. Both significantly improved following surgical treatment and remained almost unchanged over the time $(* P<0.05$ versus pre-operative period). No statistical difference was observed among the postoperative evaluations
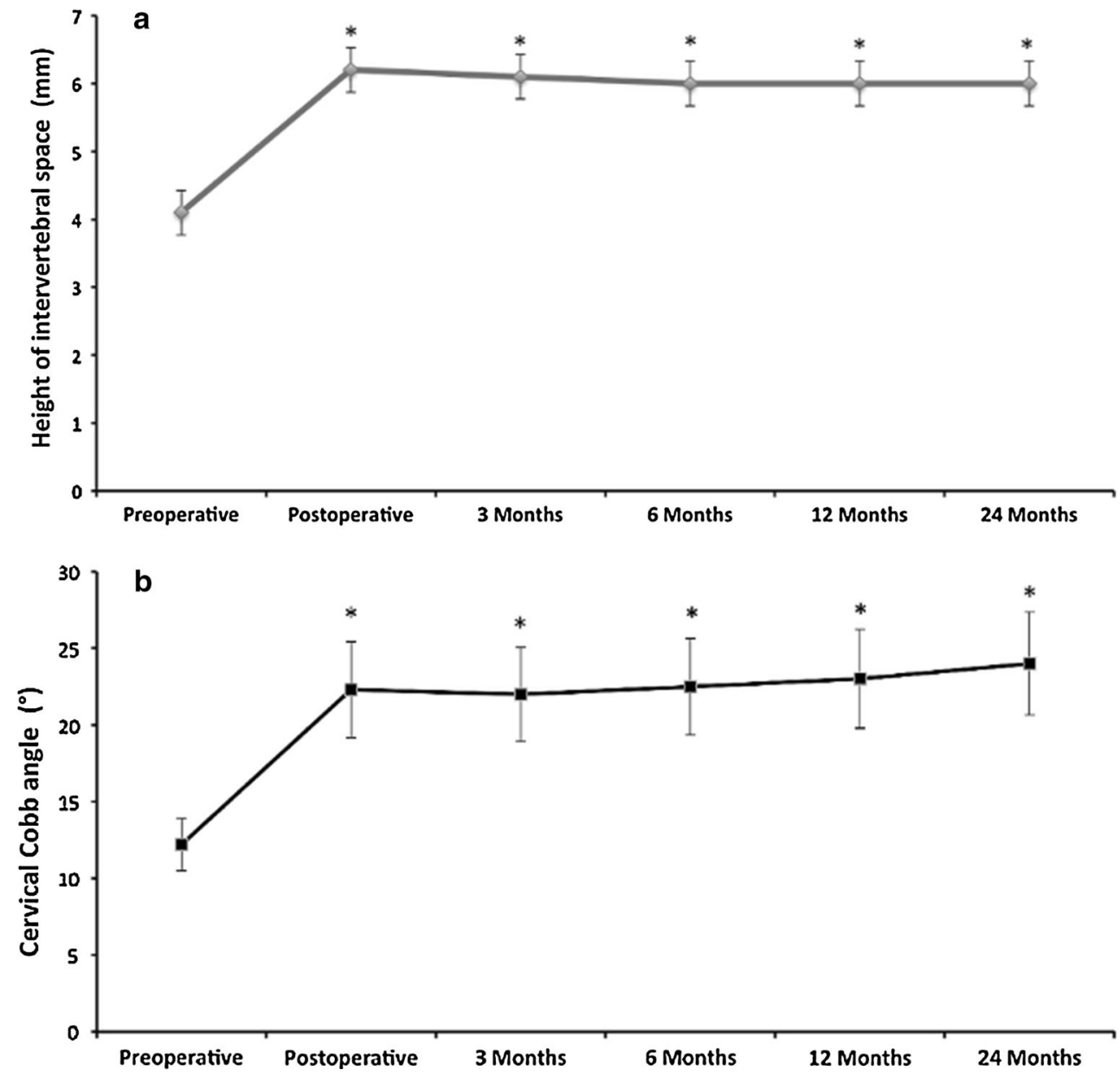

device subsidence or segmental collapse by 24 months; there were no evidences of cage mobilization or dislodgement or of mobilization of the anchoring system in both vertebral bodies. Finally, no evidence of progressive kyphosis, heterotopic bone formation, pseudarthrosis, or development of adjacent-level degeneration was reported.

Fusion was observed in all the cases (100\%) in each operated segment according to the criteria suggested by Pitzen and collaborators [8]. Cervical radiography or CT scan well revealed such findings. No cases of subsidence have been seen since the two wings of the cage allowed a firm cage allocation.

The height of intervertebral space was significantly improved from $4.1 \pm 0.7 \mathrm{~mm}$ measured before surgery to $6.2 \pm 0.4 \mathrm{~mm}$ at 1 week and $6.0 \pm 0.3 \mathrm{~mm}$ at 24 months following the surgical treatment $(P<0.05)$ (Fig. 2a). The cervical Cobb angle was also significantly improved from $12.2 \pm 4.3^{\circ}$, measured before surgery, to $22.3 \pm 3.1^{\circ}$ at 1 week and $21.1 \pm 4.1^{\circ}$ at 24 months following the implant $(P<0.05)$. No statistical differences were observed among the post-operative evaluations $(P>0.05)$ (Fig. 2b).

All patients had a reduction in both VAS neck pain and radicular arm pain $(P<0.05$ compared to pre-op)
(Fig. 3a). We observed a statistically significant reduction in NPAD-D $(P<0.001)$ (Fig. 3b) within the first 3 months compared to pre-operative baseline. We observed no statistical change neither in VAS neck and radicular arm pain, nor in NPAD-D score comparing 3, 612 and 24 months' follow-up.

\section{Illustrative case}

\section{History}

This 51-year-old woman, otherwise healthy, presented with left upper-extremity radicular pain extending into the hands with associated paresthesia. She was evaluated and treated by physical therapy without improvement.

\section{Examination}

The patient had +1 reflexes in the left biceps and left triceps, left hand paresthesia in the first three digits, a positive Spurling test for pain in the shoulders and extending into the hands, and was Grade 4/5 strength in the left biceps. 
Fig. 3 Bar graphs showing a neck pain and radicular arm pain (0-100 VAS score) and b NPAD-D pre-operatively and during follow-up $(* P<0.05$ versus pre-operative period). All patients had a reduction in both VAS, neck pain and radicular arm pain, and NPAD-D score within the first 3 months. This observation unchanged over the time
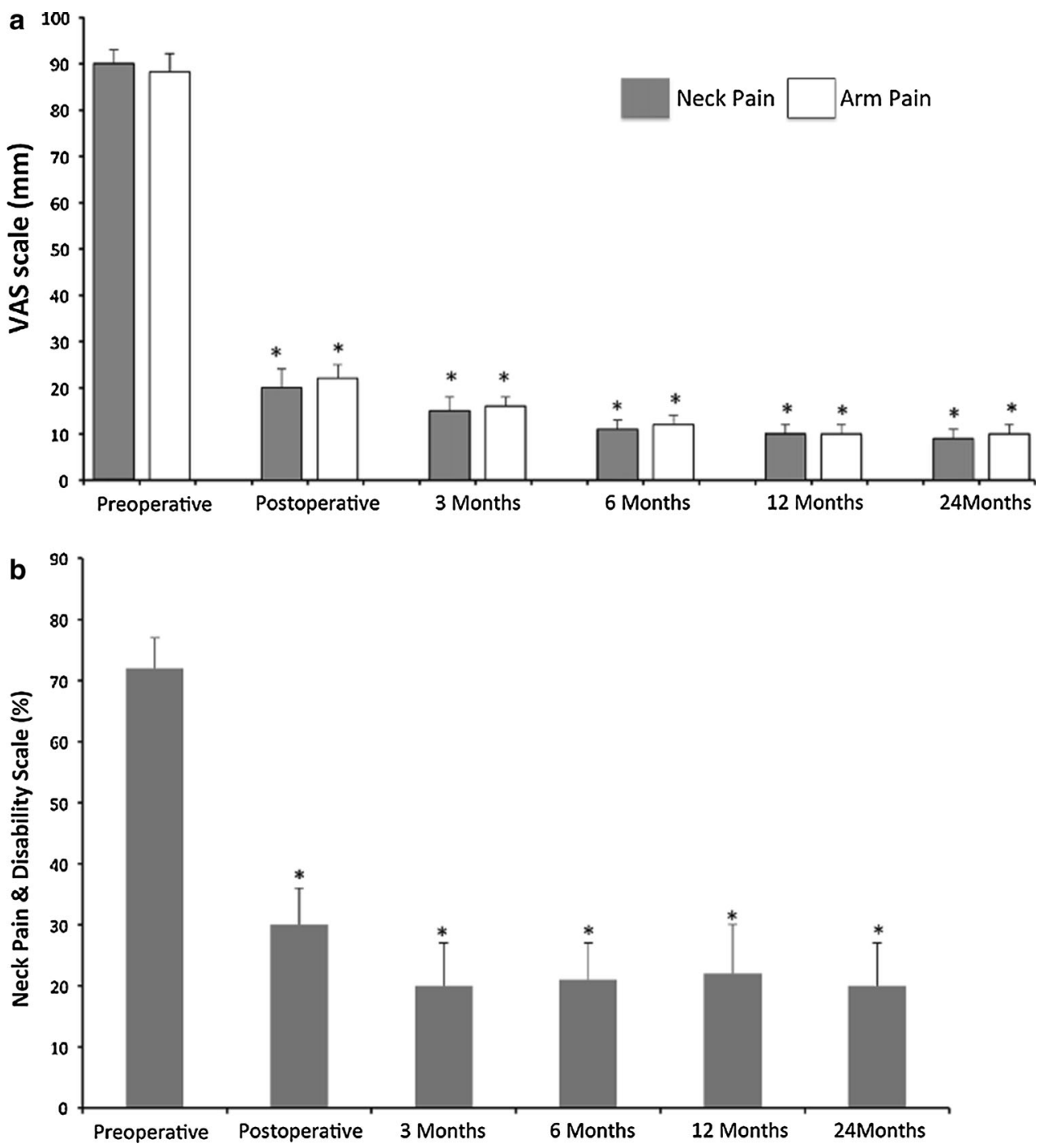

Plain radiography showed a reduction in the cervical lordosis and early degenerative disc disease at C5-C6 (Fig. 4a). Analysis of the MR images revealed C5-C6 disc herniation with left foraminal stenosis with a herniated fragment in strict contact with the radicular structure (Fig. 4b, c).

\section{Operation}

The patient underwent an anterior C5-C6 decompression. The disc was removed through classical discectomy and the left C6 root was completely decompressed. The arthrodesis was accomplished through implantation of a $14 \times 14$ and height 6-mm ROI-C cage. No intraoperative complication occurred. Intraoperative $\mathrm{X}$-ray control revealed a good cage positioning and a convenient improvement of the lordosis.

\section{Post-operative course}

The post-operative course was uneventful. All the neurological deficits recorded before the surgical treatment gradually disappeared. Neck X-ray revealed excellent placement of the device. The patient, therefore, was discharged and at 6 weeks follow-up examination, the patient stated that radicular symptoms had completely resolved. Radiographic control showed the good position of the cage and a satisfactory alignment of the cervical spine (Fig. 5).

\section{Discussion}

The reason for the development of alternative stabilization device in ACDF is avoidance of complications arising from the use of autologous or allergenic bone grafting. Use of 

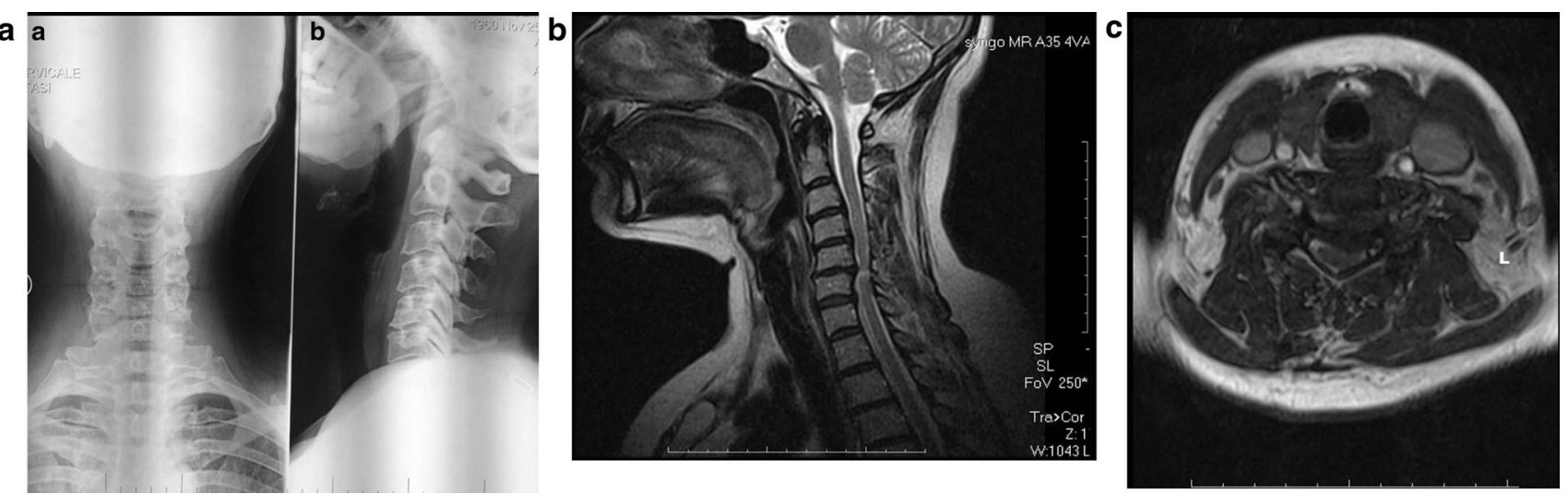

Fig. 4 Pre-operative examination. a Plain radiography showed reduction in the cervical lordosis and early degenerative disc disease at C5-C6; analysis of the MR images revealed C5-C6 disc herniation
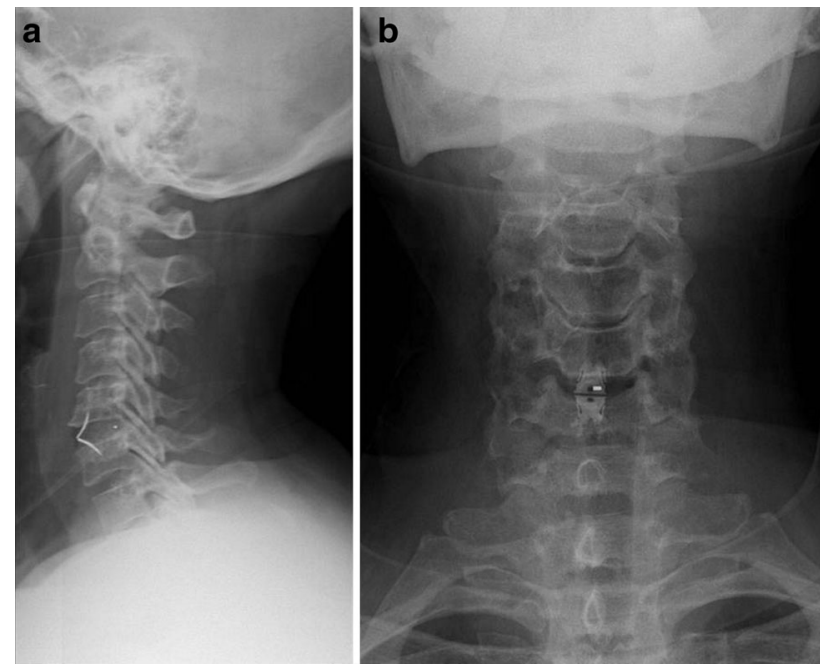

Fig. 5 Six weeks post-operative ROI-C radiographs showing good position of the cage at C5-C6 level and a satisfactory alignment of the cervical spine (a lateral view, b anterior-posterior view)

anterior iliac bone graft for anterior interbody fusion has been the gold standard for decades. Although highly successful fusion is achieved by autologous iliac bone graft, various studies have documented iliac donor site complications $[9,10]$. To prevent these complications, cages have been studied and applied in humans as potential bone substitutes for autograft in interbody fusion. The criteria required for an ideal cage for cervical interbody fusion are the following: providing immediate stability, maintaining spinal alignment and foraminal height, achieving higher or at least equal fusion success rate, and obviating complications using autograft. Titanium or carbon fiber cages were widely used for cervical interbody fusion, but subsidence, migration, and structure failure have occurred [11, 12]. Polyetheretherketone (PEEK) is a non-absorbable with left foraminal stenosis with a herniated fragment in strict contact with the radicular structure (b sagittal T2-weighted image; $\mathbf{c}$ axial T2weighted image)

biopolymer that has been used in a variety of industries including medical devices. The PEEK cages are biocompatible, radiolucent, and have modulus of elasticity similar to the bone [13].

When dealing with a patient affected by cervical spine disc disease, ACDF and cervical arthroplasty are two possible operative techniques [14-16]. According to the literature [17], only $43 \%$ of patients met the indications for cervical total disc arthroplasty. For the remaining $57 \%$ of suffering patients, ACDF is still the gold standard to achieve the reduction of the neurological deficits.

The present study is the first objectively analyzing prospectively the use of ROI-C cages in ACDF. The results indicate that the use of this PEEK cage is safe in standard ACDF procedure even if a longer follow-up is needed. Interestingly, a thorough search of the world literature revealed no peer-reviewed journal article on the use of the ROI-C cage in ACDF to date. Therefore, the findings reported here represents, to our knowledge, the only information supporting this device. It appears to be acceptably safe, at least in medium-term follow-up.

One of the most reported complications following ACDF is chronic dysphagia that can reach $21 \%$ in some studies [18]. For the early post-operative period, the rate of dysphagia in our study was lower than what reported in the current literature $[6,19]$. Only 2 of 32 patients complained about mild symptoms of dysphagia, and only one patient was stable at 6 months follow-up with very mild dysphagia with a complete resolution at 24 months follow-up. Although the exact pathophysiological mechanisms underlying postoperative dysphagia remain unknown, post-operative soft tissue oedema, oesophageal injury, post-operative hematoma, and adhesive formations around implanted cervical plates might be possible explanations for such a symptom [20]. Some authors have advocated a strict correlation between the device's thickness and dysphagia rate [21]. Cage 
with very low profile avoids an implant contact to the soft tissue in front of the cervical spine. This might avoid any mechanical irritation of the esophagus and may explain the low dysphagia rate in our patients.

Cervical arthrodesis is a well-known surgical procedure. Favorable outcomes and the prospect of a lower incidence of adjacent-level disease have encouraged expanding current indications to include multilevel disc disease [22-25]. According to previous studies considering different cages, ROI-C offered fusion in all the cases and prevented from subsidence to be observed [26]. We consider that the two wings firmly introduced in the vertebral bodies can provide initial stability until the fusion time. We found that the intervertebral height was significantly increased from $4.1 \pm 0.7$ to $6 \pm 0.3 \mathrm{~mm}$ at 24 months following surgery $(P<0.05)$, and the cervical Cobb angle was significantly improved from $12.2 \pm 4.3^{\circ}$ at pre-operation to $21.1 \pm 4.1^{\circ}$ at 24 months follow-up $(P<0.05)$.

The study suffers of some limitations. First, the study has been conducted as an observational study without a control group. Second, the follow-up was short to assume firm observations.

Overall, our results suggest that cervical ROI-C system is a safe and effective device for decompression in patients with cervical radiculopathy and myelopathy. Main advantages of the prosthesis are zero profile and self-locking plates designed for initial stability and warrant future complete fusion. The cage has demonstrated to offer high rate of fusion, restoration of the cervical lordosis and disc space height. Long-term follow-up is necessary to evaluate any potential advantage over multilevel cervical fusions in regard to adjacent-level disease.

\section{Conclusions}

Cervical arthrodesis by ROI-C system has demonstrated to be a safe and effective alternative to many other devices. It incorporates a different philosophy of insertion besides the guarantee of fusion. Data from our study might encourage its use for the management of cervical radiculopathy and myelopathy.

Acknowledgments The study, in part, has been supported by the Academic funds (ex $60 \%$ of the first author).

Conflict of interest The authors report no declarations of interest.

\section{References}

1. Zaveri GR, Ford M (2001) Cervical spondylosis: the role of anterior instrumentation after decompression and fusion. J Spinal Disord 14:10-16
2. Bijur PE, Silver W, Gallagher EJ (2001) Reliability of the visual analog scale for measurement of acute pain. Acad Emerg Med 8:1153-1157

3. Carlsson A (1983) Assessment of chronic pain. I. Aspects of the reliability and validity of the visual analogue scale. Pain $16: 87-101$

4. Goolkasian P, Wheeler A, Gretz S (2002) The neck pain and disability scale: test-retest reliability and construct validity. Clin J Pain 18:245-250

5. Scherer M, Blozik E, Himmel W, Laptinskaya D, Kochen MM, Herrmann-Lingen C (2008) Psychometric properties of a German version of the neck pain and disability scale. Eur Spine $\mathrm{J}$ 17:922-929

6. Bazaz R, Lee MJ, Yoo JU (2002) Incidence of dysphagia after anterior cervical spine surgery: a prospective study. Spine 27:2453-2458

7. Zdeblick TA, Ducker TB (1991) The use of freeze-dried allograft bone for anterior cervical fusions. Spine 16:726-729

8. Pitzen TR, Chrobok J, Stulik J, Ruffing S, Drumm J, Sova L, Kucera R, Vyskocil T, Steudel WI (2009) Implant complications, fusion, loss of lordosis, and outcome after anterior cervical plating with dynamic or rigid plates: two-year results of a multicentric, randomized, controlled study. Spine 34:641-646

9. Arrington ED, Smith WJ, Chambers HG, Bucknell AL, Davino NA (1996) Complications of iliac crest bone graft harvesting. Clin Orthop 329:300-309

10. Goulet JA, Senunas LE, DeSilva GL, Greenfield ML (1997) Autogenous iliac crest bone graft. Complications and functional assessment. Clin Orthop 339:76-81

11. Niu CC, Chen LH, Lai PL, Fu TS, Chen WJ (2005) Trapezoidal titanium cage in anterior cervical interbody fusion: a clinical experience. Chang Gung Med J 28:212-221

12. van der Haven I, van Loon PJ, Bartels RH, van Susante JL (2005) Anterior cervical interbody fusion with radiolucent carbon fiber cages: clinical and radiological results. Acta Orthop Belg 71:604-609

13. Cho DY, Lee WY, Sheu PC, Chen CC (2005) Cage containing a biphasic calcium phosphate ceramic (Triosite) for the treatment of cervical spondylosis. Surg Neurol 63:497-503 (discussion 503-494)

14. Nabhan A, Ahlhelm F, Pitzen T, Steudel WI, Jung J, Shariat K, Steimer O, Bachelier F, Pape D (2007) Disc replacement using Pro-disc $\mathrm{C}$ versus fusion: a prospective randomised and controlled radiographic and clinical study. Eur Spine J 16:423-430

15. Sasso RC, Smucker JD, Hacker RJ, Heller JG (2007) Clinical outcomes of BRYAN cervical disc arthroplasty: a prospective, randomized, controlled, multicenter trial with 24-month followup. J Spinal Disord Tech 20:481-491

16. Wang Y, Cai B, Zhang XS, Xiao SH, Wang Z, Lu N, Chai W, Zheng GQ (2008) Clinical outcomes of single level Bryan cervical disc arthroplasty: a prospective controlled study. Zhonghua Wai Ke Za Zhi 46:328-332

17. Auerbach JD, Jones KJ, Fras CI, Balderston JR, Rushton SA, Chin KR (2008) The prevalence of indications and contraindications to cervical total disc replacement. Spine J 8:711-716

18. Riley LH 3rd, Skolasky RL, Albert TJ, Vaccaro AR, Heller JG (2005) Dysphagia after anterior cervical decompression and fusion: prevalence and risk factors from a longitudinal cohort study. Spine 30:2564-2569

19. Yue WM, Brodner W, Highland TR (2005) Persistent swallowing and voice problems after anterior cervical discectomy and fusion with allograft and plating: a 5- to 11-year follow-up study. Eur Spine J 14:677-682

20. Fountas KN (2005) Anterior cervical fusion using dense cancellous allografts and dynamic plating. Neurosurgery 56:E1166 (author reply E1166) 
21. Lee M, Bazaz R, Furey C, Yoo J (2005) Influence of anterior cervical plate design on dysphagia: a 2-year prospective longitudinal follow-up study. J Spinal Disord Tech 18:406-409

22. Gore DR (2001) The arthrodesis rate in multilevel anterior cervical fusions using autogenous fibula. Spine 26:1259-1263

23. Resnick DK, Trost GR (2007) Use of ventral plates for cervical arthrodesis. Neurosurgery 60:S112-S117

24. Shin DA, Yi S, Yoon do H, Kim KN, Shin HC (2009) Artificial disc replacement combined with fusion versus two-level fusion in cervical two-level disc disease. Spine 34:1153-1159 (discussion 1160-1151)
25. Hilibrand AS, Carlson GD, Palumbo MA, Jones PK, Bohlman HH (1999) Radiculopathy and myelopathy at segments adjacent to the site of a previous anterior cervical arthrodesis. J Bone Jt Surg 81:519-528

26. Kim SJ, Kim SD (2014) Anterior cervical discectomy and fusion using a double cylindrical cage versus an anterior cervical plating system with Iliac crest autografts for the treatment of cervical degenerative disc disease. J Korean Neurosurg Soc 55:12-17. doi:10.3340/jkns.2014.55.1.12 